\title{
Variação da entropia total para um corpo em contato com reservatórios térmicos: o caminho da reversibilidade
}

Total entropy variation for an object in contact with heat reservoirs: the path to reversibility

\author{
Lucas R. D. Freitas ${ }^{1}$, Luiz Felipe C. Pereira*1] \\ ${ }^{1}$ Universidade Federal do Rio Grande do Norte, Departamento de Física, Natal, RN, Brasil
}

\begin{abstract}
Recebido em 28 de Novembro de 2018. Revisado em 07 de Abril de 2019. Aceito em 16 de Abril de 2019
\end{abstract}
\begin{abstract}
A segunda lei da termodinâmica é um dos tópicos de física fundamental menos compreendidos por estudantes e profissionais de ciências exatas e engenharias, talvez devido à sua sutileza e abundância de enunciados. Neste trabalho analisamos a variação de entropia de um sistema isolado, formado por um corpo de capacidade calorífica $C(T)$ e um ou mais reservatórios térmicos, quando a temperatura absoluta do corpo varia através da troca de calor com os reservatórios de maneira sucessiva. Obtemos uma expressão geral para a variação de entropia total, $\Delta S^{(0)}$, em função de $C(T)$ e do número de reservatórios térmicos $N$. Mostramos numericamente que $\Delta S^{(0)}$ diminui à medida de $N$ aumenta e, considerando que no limite $N \rightarrow \infty$ a diferença de temperatura entre reservatórios sucessivos é infinitesimal, mostramos analiticamente que $\lim _{N \rightarrow \infty} \Delta S^{(0)}=0$, resultado esperado da segunda lei da termodinâmica para um processo quase-estático reversível. Por fim, sugerimos que a demonstração de que a variação da entropia total é nula quando a temperatura do corpo varia quase-estaticamente pode servir de base para uma avaliação do entendimento da segunda lei da termodinâmica por parte de estudantes de graduação em ciências exatas e engenharias.
\end{abstract}

Palavras-chave: segunda lei da termodinâmica, transferência de calor, variação de entropia.

\begin{abstract}
The second law of thermodynamics is one of the least understood fundamental physical laws, even among science and engineering students and professionals, possibly due to its subtleness and several seemingly different statements. Here we investigate the entropy variation of an isolated system composed of an object of heat capacity $C(T)$ and one or more heat reservoirs, as the absolute temperature of the object varies due to heat exchange with subsequent reservoirs. We obtain a general expression for the total entropy variation, $\Delta S^{(0)}$, in terms of $C(T)$ and the number of reservoirs $N$. We numerically show that $\Delta S^{(0)}$ decreases as $N$ increases, and considering that as $N \rightarrow \infty$ the temperature difference between subsequent reservoirs becomes infinitesimal, we analytically show that $\lim _{N \rightarrow \infty} \Delta S^{(0)}=0$, in accordance with the second law of thermodynamics for a reversible quasi-static process. We conclude by proposing an undergraduate exam problem based on the demonstration that the total entropy variation vanishes in the quasi-static limit.
\end{abstract}

Keywords: second law of thermodynamics, heat transfer, entropy variation.

\section{Introdução}

Na sua clássica obra As duas culturas, C. P. Snow reconta anedoticamente situações em que pessoas consideradas cultas e educadas criticam a falta de cultura dos cientistas, mas quando questionadas se seriam capazes de descrever a segunda lei da termodinâmica demonstram ignorância sobre o tema [1. Na sequência, Snow argumenta que conhecer a segunda lei da termodinâmica seria tão importante quanto conhecer ao menos uma das obras de Shakespeare, e defende que a cultura científica é pelo menos tão importante quanto a cultura literária. No entanto, mesmo entre cientistas, é possível argumentar que Snow escolheu um tópico científico particularmente sutil para apresentar seu argumento. De fato, a segunda lei da termodinâmica é possivelmente um dos tópicos de

*Endereço de correspondência: pereira@fisica.ufrn.br física fundamental menos compreendidos por estudantes e profissionais das áreas de ciências exatas e engenharias.

A sutileza da segunda lei da termodinâmica fica explícita quando consideramos a abundância de seus enunciados encontrados em livros-texto de física básica [2 4], termodinâmica [5 6] e física estatística [7 11].

Por exemplo, o enunciado de Kelvin afirma que não existe processo termodinâmico cíclico em que calor é extraído de um reservatório térmico e convertido em trabalho integralmente. O enunciado atribuído a Clausius por Kelvin afirma que não é possível transferir calor de um corpo mais frio para um mais quente sem realizar trabalho sobre esse sistema, ou seja, não é possível construir um refrigerador que opere sem consumir energia. Por fim, temos o enunciado de Clausius, que utilizaremos aqui, proposto após o desenvolvimento da termodinâmica e da introdução da idéia de entropia, que afirma que em um 
sistema fechado a entropia aumenta ou permanece constante, mas nunca diminui. Alguns aspectos fundamentais da segunda lei da termodinâmica ainda são abordados em publicações recentes, como por exemplo de Oliveira 12 e Dourado e Marchiori [13], explicitando ainda mais seu caráter peculiar.

A segunda lei da termodinâmica pode ser expressa sucintamente na forma

$$
\Delta S^{(0)} \geq 0
$$

onde o sobrescrito ${ }^{(0)}$ indica que se trata de um sistema isolado. A desigualdade é válida quando o processo que leva à variação da entropia é irreversível e a igualdade é satisfeita para processos reversíveis. Processos que ocorrem de forma abrupta, onde o estado do sistema muda bruscamente, tendem a ser irreversíveis, enquanto processos que ocorrem de maneira suave, onde os estados do sistema evoluem lentamente podem ser idealmente reversíveis. Um processo quase-estático é um processo onde um sistema vai lentamente de um estado inicial de equilíbrio a um estado final de equilíbrio, passando por uma série de estados intermediários de equilíbrio, de maneira que o sistema encontra-se sempre em um estado de equilíbrio. Um reservatório de calor é um sistema que pode fornecer ou absorver calor indefinidamente, sem que sua temperatura sofra alterações. A capacidade calorífica (também chamada de capacidade térmica) é definida como a razão entre o calor recebido (ou perdido) e o aumento (ou diminuição) da temperatura de um corpo, e pode-se afirmar que a capacidade calorífica de um reservatório térmico é infinita. Em geral a capacidade calorífica é uma função da temperatura, mas existem muitos casos em que esta grandeza é aproximadamente constante.

Calkin e Kiang analisaram a variação de entropia para um corpo de capacidade calorífica constante em contato com um ou mais reservatórios térmicos sucessivamente, mostrando que a variação de entropia se anula quando o número de reservatórios tende ao infinito [14]. Thomsen e Bers analisaram a relação entre produção de entropia e reversibilidade no mesmo tipo de sistema 15. Stilck e Brum generalizaram a análise de Calkin e Kiang para o caso de um corpo com capacidade calorífica dependente da temperatura, e mostraram que a variação de entropia é inversamente proporcional ao número de reservatórios quando o número de reservatórios é grande [16]. Lima, e posteriormente Freire e Leite, analisaram a variação de entropia na transferência de calor entre dois corpos distintos de capacidades caloríficas constantes, concluindo que a variação de entropia é de fato positiva, satisfazendo a segunda lei da termodinâmica 17,18.

Neste trabalho abordamos a segunda lei da termodinâmica analisando a variação de entropia de um sistema isolado, formado por um corpo de capacidade calorífica $C(T)$ e um ou mais reservatórios térmicos, quando a temperatura absoluta do corpo varia de um valor inicial $T_{i}$ a um valor final $T_{f}$ através da troca de calor com os reservatórios de maneira sucessiva. A cada estágio é necessário aguardar que o corpo esteja em equilíbrio térmico com o reservatório, ou seja, que sua temperatura seja igual à do reservatório, antes de prosseguir para o próximo reservatório. Mantendo o intervalo $\left[T_{i}, T_{f}\right]$ fixo, quanto maior for o número de reservatórios $N$ menor será a diferença de temperatura entre reservatórios sucessivos, e à medida que $N$ aumenta o processo aproxima-se do limite quase-estático. Considerando a variação de entropia do corpo e dos reservatórios, obtemos uma expressão geral para a variação de entropia total, $\Delta S^{(0)}$, em função de $C(T)$ e do número de reservatórios térmi$\cos N$. Mostramos numericamente que $\Delta S^{(0)}$ diminui à medida que $N$ aumenta e, considerando que no limite $N \rightarrow \infty$ a diferença de temperatura entre reservatórios sucessivos é infinitesimal, mostramos analiticamente que $\lim _{N \rightarrow \infty} \Delta S^{(0)}=0$, resultado esperado da segunda lei da termodinâmica para um processo quase-estático reversível. Na seção 5 argumentamos que a demonstração de que a variação da entropia total é nula quando a temperatura do corpo varia quase-estaticamente pode servir de base para uma avaliação do entendimento da segunda lei da termodinâmica por parte de estudantes de graduação nas áreas de ciências exatas e engenharias.

\section{Reservatório térmico único}

Consideramos o corpo sendo um sistema $A$, o reservatório térmico $A^{\prime}$ e o sistema isolado formado pelos dois: $A^{(0)}=$ $A+A^{\prime}$, tal que a variação da entropia total é dada por

$$
\Delta S^{(0)}=\Delta S+\Delta S^{\prime}
$$

A variação de entropia do corpo $A$ depende apenas da sua temperatura inicial e final e pode ser calculada a partir de $d S=d Q / T$, tal que

$$
\Delta S=\int_{i}^{f} \frac{d Q}{T}=\int_{T_{i}}^{T_{f}} \frac{C(T) d T}{T},
$$

onde $C(T)$ é a capacidade calorífica do corpo. Enquanto isso, a variação de entropia do reservatório de calor $A^{\prime}$ é

$$
\Delta S^{\prime}=\int \frac{d Q^{\prime}}{T^{\prime}}=\frac{1}{T_{f}} \int d Q^{\prime}=\frac{Q^{\prime}}{T_{f}}
$$

onde $Q^{\prime}$ é o calor recebido pelo reservatório e $T_{f}$ é a sua temperatura absoluta. Sendo $A^{(0)}$ um sistema isolado, sabemos que $Q+Q^{\prime}=0$, então

$$
Q^{\prime}=-Q=-\int_{T_{i}}^{T_{f}} C(T) d T .
$$

Logo

$$
\Delta S^{\prime}=-\int_{T_{i}}^{T_{f}} \frac{C(T)}{T_{f}} d T,
$$

onde lembramos que $T_{f}$, a temperatura absoluta do reservatório, é constante. 
Finalmente, a variação da entropia total é

$$
\Delta S^{(0)}=\int_{T_{i}}^{T_{f}} \frac{C(T)}{T} d T-\int_{T_{i}}^{T_{f}} \frac{C(T)}{T_{f}} d T .
$$

Sendo $T \leq T_{f}$ sabemos que o segundo termo é menor que o primeiro tal que $\Delta S^{(0)}>0$, satisfazendo a segunda lei da termodinâmica para um processo irreversível.

\section{Vários reservatórios térmicos em sucessão}

Consideramos agora que o corpo $A$ é colocado sucessivamente em contato com uma série de $N$ reservatórios térmicos, com valores de temperatura igualmente espaçados.

A cada estágio é necessário aguardar que o corpo esteja em equilíbrio térmico com o reservatório antes de prosseguir para o próximo reservatório.

A temperatura do $n$-ésimo reservatório pode ser escrita como

$$
T_{n}=T_{i}+n\left(\frac{T_{f}-T_{i}}{N}\right)=T_{i}+n(\Delta T)
$$

com $n=1,2, \cdots, N$.

A variação de entropia de cada reservatório será

$$
\Delta S_{n}^{\prime}=-\int_{T_{n-1}}^{T_{n}} \frac{C(T)}{T_{n}} d T
$$

tal que a variação de entropia considerando todos os reservatórios é dada por

$$
\Delta S^{\prime}=\sum_{n=1}^{N} \Delta S_{n}^{\prime}=-\sum_{n=1}^{N} \int_{T_{n-1}}^{T_{n}} \frac{C(T)}{T_{n}} d T
$$

que é uma relação exata.

Finalmente, a variação da entropia total fica

$$
\Delta S^{(0)}=\int_{T_{i}}^{T_{f}} \frac{C(T)}{T} d T-\sum_{n=1}^{N} \int_{T_{n-1}}^{T_{n}} \frac{C(T)}{T_{n}} d T .
$$

De posse da equação (11), podemos agora verificar numericamente se a descrição acima está de acordo com a segunda lei da termodinâmica.

\subsection{Caso $C(T)=C$}

Tomemos, inicialmente, um sistema simples cuja capacidade calorífica seja aproximadamente independente da temperatura no intervalo considerado, como por exemplo a água em temperaturas da ordem de $20{ }^{\circ} \mathrm{C}$ ou um sólido acima da sua temperatura de Debye.

Neste caso, $C(T)=C$ e a equação (11) fica

$$
\Delta S^{(0)}=C \int_{T_{i}}^{T_{f}} \frac{d T}{T}-\sum_{n=1}^{N} \frac{C}{T_{n}} \int_{T_{n-1}}^{T_{n}} d T .
$$

Realizando as duas integrações obtemos

$$
\Delta S^{(0)}=C\left[\ln \frac{T_{f}}{T_{i}}-\sum_{n=1}^{N} \frac{\Delta T}{T_{n}}\right] .
$$

Em princípio, a expressão acima pode ser utilizada para obter valores de $\Delta S^{(0)}$ em função da capacidade calorífica constante $C$ para qualquer intervalo de temperaturas finitas $T_{i} \leq T \leq T_{f}$. Vamos considerar, por exemplo, uma situação onde $T_{f}=2 T_{i}$, tal que $\Delta T=T_{i} / N$ e $T_{n}=T_{i}(N+n) / N$. Neste caso, a expressão fica

$$
\Delta S^{(0)}=C\left[\ln 2-\sum_{n=1}^{N} \frac{1}{n+N}\right] .
$$

Esta expressão pode ser utilizada para obter valores numéricos para $\Delta S^{(0)} / C$ para qualquer valor de $N$ com uma calculadora científica, uma planilha eletrônica, ou uma simples rotina numérica implementada em linguagem de programação como $\mathrm{C}$ ou Python. A Tabela 1 apresenta alguns valores calculados a partir da equação (14).

Na Figura 1 mostramos o comportamento de $\Delta S^{(0)} / C$ em função de $1 / N$ para valores de $N=10^{2} \cdots 10^{6}$, obtidos com a expressão acima (círculos na figura). A escolha de $1 / N$ ao invés de $N$ permite uma extrapolação direta para o limite $N \rightarrow \infty$, já que este corresponde à origem do gráfico, $1 / N \rightarrow 0$. A linha tracejada apresenta um ajuste linear por mínimos quadrados para os pontos obtidos pela rotina numérica considerando apenas $N \geq 100$. Observe que no limite $1 / N \rightarrow 0$ temos $\Delta S^{(0)} / C \rightarrow 0$, como esperado da segunda lei da termodinâmica para um processo quase-estático reversível. No Apêndice A apresentamos uma derivação analítica do coeficiente angular da reta na Figura 1

\subsection{Caso $C(T)=c_{0} T^{r}$}

Vamos considerar agora um segundo caso, onde a capacidade calorífica do corpo depende da temperatura na forma de uma lei de potência $C(T)=c_{0} T^{r} \operatorname{com} r>0$ no intervalo de temperaturas finitas $T_{i} \leq T \leq T_{f}$. Observe que este comportamento está de acordo com a terceira lei da termodinâmica, já que $C(T) \rightarrow 0$ no limite de zero absoluto, e descreve adequadamente metais $(r=1)$ e isolantes $(r=3)$ [7].

Tabela 1: Valores para a variação da entropia total em função do número de reservatórios obtidos das equações (14) e (17) para $r=1$ e $3 . \Delta S^{(0)}$ diminui à medida que $N$ aumenta.

\begin{tabular}{cccc}
\hline $\mathrm{N}$ & $\Delta S^{(0)} / C$ & $\Delta S^{(0)} / c_{o} T_{i}^{r=1}$ & $\Delta S^{(0)} / c_{o} T_{i}^{r=3}$ \\
\hline 1 & $1.93 \times 10^{-1}$ & $2.50 \times 10^{-1}$ & $4.58 \times 10^{-1}$ \\
2 & $1.10 \times 10^{-1}$ & $1.46 \times 10^{-1}$ & $2.89 \times 10^{-1}$ \\
5 & $4.75 \times 10^{-2}$ & $6.46 \times 10^{-2}$ & $1.35 \times 10^{-1}$ \\
10 & $2.44 \times 10^{-2}$ & $3.34 \times 10^{-2}$ & $7.10 \times 10^{-2}$ \\
100 & $2.49 \times 10^{-3}$ & $3.45 \times 10^{-3}$ & $7.46 \times 10^{-3}$ \\
10000 & $2.50 \times 10^{-5}$ & $3.47 \times 10^{-5}$ & $7.50 \times 10^{-5}$ \\
1000000 & $2.50 \times 10^{-7}$ & $3.47 \times 10^{-7}$ & $7.50 \times 10^{-7}$ \\
\hline
\end{tabular}




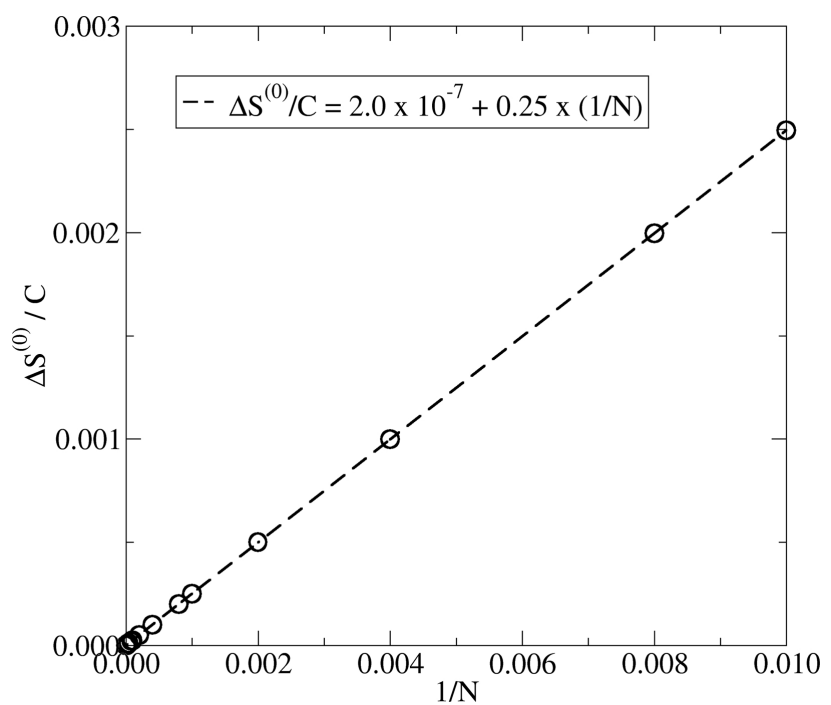

Figura 1: Variação da entropia total em função do inverso do número de reservatórios térmicos $1 / N$. Os pontos são obtidos da equação (14). A linha tracejada é um ajuste linear por mínimos quadrados considerando apenas $N \geq 100$. No limite $N \rightarrow \infty$ vemos que $\Delta S^{(0)} / C \rightarrow 0$, como esperado da segunda lei da termodinâmica para um processo quase-estático reversível.

Neste caso, a equação 11] fica

$$
\Delta S^{(0)}=\int_{T_{i}}^{T_{f}} \frac{c_{0} T^{r}}{T} d T-\sum_{n=1}^{N} \int_{T_{n}-1}^{T_{n}} \frac{c_{0} T^{r}}{T_{n}} d T .
$$

Integrando ambos os termos, ficamos com

$$
\Delta S^{(0)}=c_{0}\left[\frac{T_{f}^{r}-T_{i}^{r}}{r}\right]-\sum_{n=1}^{N} \frac{c_{0}}{T_{n}}\left[\frac{T_{n}^{r+1}-T_{n-1}^{r+1}}{r+1}\right] .
$$

Considerando novamente o caso especial $T_{f}=2 T_{i}$, tal que $\Delta T=T_{i} / N$ e $T_{n}=T_{i}(N+n) / N$, obtemos então

$$
\begin{aligned}
& \Delta S^{(0)}=c_{0} T_{i}^{r}\left[\frac{2^{r}-1}{r}-\right. \\
& \left.\frac{1}{r+1} \sum_{n=1}^{N} \frac{(N+n)^{r+1}-(N+n-1)^{r+1}}{(N+n) N^{r}}\right]
\end{aligned}
$$

Lembrando que $c_{0} T_{i}^{r}=C\left(T_{i}\right)$, podemos agora fazer uma análise numérica do comportamento de $\Delta S^{(0)} / C\left(T_{i}\right)$ no limite $N \rightarrow \infty$ para vários valores de $r$. A Tabela 1 apresenta alguns valores calculados a partir da equação (17) para os casos $r=1$ e $r=3$. Na Figura 2 as linhas tracejadas são ajustes lineares por mínimos quadrados para os dados obtidos da equação 17) considerando apenas $N \geq 100$. Novamente, vemos que $\Delta S^{(0)} / C\left(T_{i}\right) \rightarrow 0$ no limite $1 / N \rightarrow 0$ para todos os valores de $r$ considerados, de acordo com o esperado da segunda lei da termodinâmica para um processo quase-estático reversível.

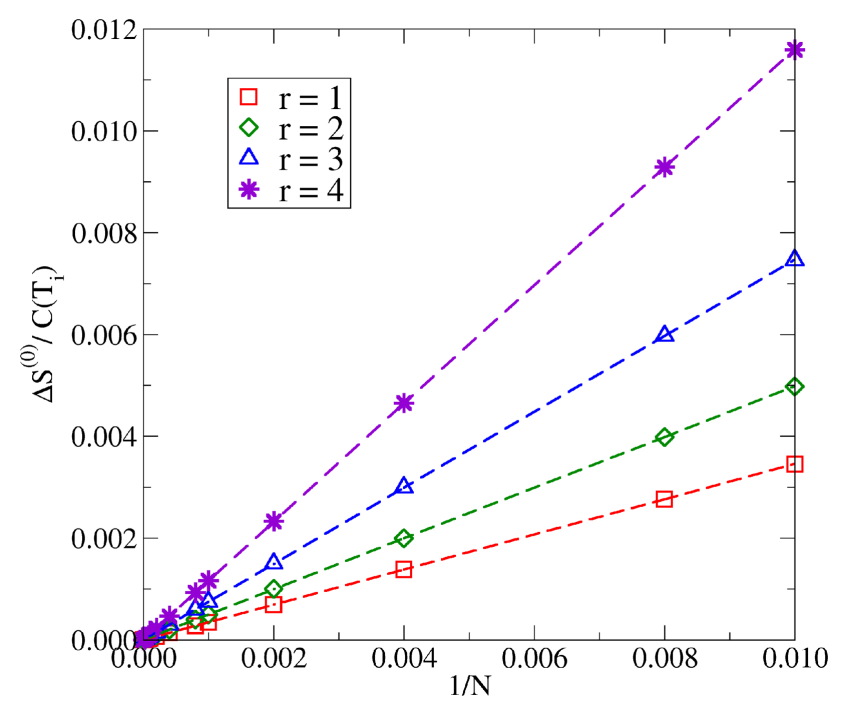

Figura 2: Variação da entropia total, em função do inverso do número de reservatórios térmicos $1 / N$ quando o calor específico do corpo é da forma $C(T)=c_{0} T^{r}$. Os pontos são obtidos da equação (17). As linhas tracejadas são ajustes lineares por mínimos quadrados para $N \geq 100$. No limite $N \rightarrow \infty$ vemos que $\Delta S^{(0)} / C\left(T_{i}\right) \rightarrow 0$ para todos os valores de $r$ considerados, como esperado da segunda lei da termodinâmica para um processo quase-estático reversível.

\section{Limite quase-estático $N \rightarrow \infty$}

As verificações numéricas apresentadas na seção anterior são uma boa indicação de que no limite $N \rightarrow \infty$ a variação de entropia total toma o valor $\Delta S^{(0)}=0$, que indica tratar-se de um processo reversível, já que a temperatura do corpo varia quase-estaticamente através do contato térmico com reservatórios de temperaturas cada vez mais próximas. No entanto, podemos ir além da verificação numérica e mostrar analiticamente que, de fato, quando o número de reservatórios é muito grande, e consequentemente a diferença de temperatura entre reservatórios subsequentes é infinitesimal, a variação da entropia total é nula. Observe que nesse limite podemos considerar $C(T)$ constante no intervalo $\Delta T=T_{n}-T_{n-1}$, tal que a integral na equação 9 pode ser escrita como

$$
\int_{T_{n-1}}^{T_{n}} \frac{C(T)}{T_{n}} d T=\frac{C\left(T_{n-1}\right) \Delta T}{T_{n}} .
$$

Agora, a somatória na equação 10 toma a forma de uma integral de Riemann,

$$
\lim _{N \rightarrow \infty} \sum_{i=1}^{N} \Delta T=\int_{T_{i}}^{T_{f}} d T
$$

de forma que a equação 10 fica escrita como

$$
\lim _{N \rightarrow \infty} \Delta S^{\prime}=-\sum_{n=1}^{\infty} \frac{C\left(T_{n-1}\right) \Delta T}{T_{n}}=-\int_{T_{i}}^{T_{f}} \frac{C(T)}{T} d T .
$$


Finalmente, da equação (11) temos

$$
\Delta S^{(0)}=\int_{T_{i}}^{T_{f}} \frac{C(T)}{T} d T-\int_{T_{i}}^{T_{f}} \frac{C(T)}{T} d T=0
$$

demonstrando que no limite $N \rightarrow \infty$ a variação de entropia total é nula já que trata-se de um processo quaseestático e reversível. É importante salientar que a transferência de calor de um reservatório térmico para um corpo sem aumento da entropia tem aplicação prática muito limitada, já que um processo quase-estático com $N \rightarrow \infty$ requer um tempo infinitamente grande para sua realização. Nosso objetivo principal neste trabalho é explorar a passagem de um processo abrupto (irreversível) quando $N$ é pequeno, para um processo quase-estático (reversível) à medida que $N$ torna-se cada vez maior. No Apêndice B mostramos que nossa análise permanece válida mesmo quando a capacidade calorífica do corpo se anula próximo ao zero absoluto.

\section{Avaliação do entendimento}

A demonstração de que a variação da entropia total é nula quando a temperatura do corpo varia quaseestaticamente pode servir de base para uma avaliação do entendimento da segunda lei da termodinâmica por parte de estudantes de graduação. Interessante notar que a questão pode ser utilizada tanto numa disciplina introdutória de termodinâmica baseada em livros-texto de física básica 2 4], quanto numa disciplina de termodinâmica e física estatística baseada em livros-texto mais avançados [5 11].

O enunciado da questão pode ser colocado como: Um corpo $A$ com capacidade calorífica $C$ e temperatura absoluta $T_{i}$ é colocado em contato térmico com um reservatório de calor $A^{\prime}$ a uma temperatura $T_{f}>T_{i}$. Quando o corpo atinge o equilíbrio térmico com o reservatório, sabemos que a temperatura absoluta de $A$ será igual a $T_{f}$. Podemos considerar que o sistema total $A^{(0)}=A+A^{\prime}$ é um sistema isolado.

- Obtenha uma expressão para a variação de entropia do corpo $A, \Delta S$, do reservatório térmico $\Delta S^{\prime}$ e do sistema fechado $\Delta S^{(0)}$, em função de $T_{i}, T_{f}$ e $C$.

- Argumente por que a expressão obtida está de acordo com a segunda lei da termodinâmica.

- Considere agora que a temperatura do corpo $A$ varia de $T_{i}$ a $T_{f}$ utilizando sucessivamente $N$ reservatórios de calor com temperaturas igualmente espaçadas. Mostre que a temperatura do n-ésimo reservatório pode ser escrita como

$$
T_{n}=T_{i}+n \frac{\Delta T}{N}
$$

onde $n=1,2 \ldots N$ e $\Delta T=T_{f}-T_{i}$.

- Obtenha uma expressão para a variação da entropia do sistema fechado $\Delta S^{(0)}$ em função de $T_{i}, T_{f}, C$, $T_{n}$ e $\Delta T$.
- Um processo quase-estático corresponde ao caso $N \rightarrow \infty$, tal que $\Delta T \rightarrow d T$. Mostre que nesse caso

$$
\lim _{N \rightarrow \infty} \Delta S^{(0)}=0 .
$$

\section{Conclusão}

A variação da entropia de um sistema isolado, formado por um corpo de capacidade calorífica $C(T)$ em contato com um ou mais reservatórios de calor, deve satisfazer a segunda lei da termodinâmica, $\Delta S^{(0)} \geq 0$, tal que a igualdade é satisfeita apenas quando a processo que leva à variação da entropia é reversível. Utilizando apenas um reservatório térmico o processo não é reversível e $\Delta S^{(0)}>0$. Considerando sucessivamente $N$ reservatórios com temperaturas igualmente espaçadas, mostramos numericamente que $\Delta S^{(0)}$ diminui à medida de $N$ aumenta, e analisando o comportamento de $\Delta S^{(0)}$ em função de $1 / N$ mostramos numericamente que $\lim _{N \rightarrow \infty} \Delta S^{(0)} \rightarrow 0$. Finalmente, considerando que no limite $N \rightarrow \infty$ a diferença de temperatura entre reservatórios sucessivos é infinitesimal, o processo torna-se quase-estático e mostramos analiticamente que $\lim _{N \rightarrow \infty} \Delta S^{(0)}=0$, de acordo com a segunda lei da termodinâmica para um processo quaseestático reversível. Por fim, sugerimos que a demonstração de que a variação da entropia total é nula quando a temperatura do corpo varia quase-estaticamente pode servir de base para uma avaliação do entendimento da segunda lei da termodinâmica por parte de estudantes de graduação nas áreas de ciências exatas e engenharias.

\section{Agradecimentos}

Agradecemos Ananias M. Mariz, F. G. Brady Moreira, Leonardo C. de Melo e Tommaso Macrì pelas críticas e sugestões. Os autores agradecem ao apoio financeiro da CAPES e do CNPq (processo 309961/2017-3). LFCP dedica este trabalho à memória do Prof. M. Howard Lee 19.

\section{Material suplementar}

O seguinte material suplementar está disponível online: Apêndice A

Apêndice B

\section{Referências}

[1] C.P. Snow, As duas culturas e uma segunda leitura (EDUSP, São Paulo, 1995).

[2] D. Halliday, R. Resnick e J.Walker, Fundamentos de física vol. 2 - Gravitação, ondas e termodinâmica (LTC, Rio de Janeiro, 2012), $9^{a} \mathrm{Ed}$.

[3] H.D. Young e R.A. Freedman, Sears \& Zemansky Física II - Termodinâmica e ondas (Pearson, São Paulo, 2008), $12^{a} \mathrm{Ed}$. 
[4] H.M. Nussenzveig, Curso de física básica: Fluidos, oscilações e ondas, calor (Blucher, São Paulo, 2014), $5^{a}$ Ed.

[5] E. Fermi, Thermodynamics (Dover, New York, 1956).

[6] M.J. de Oliveira, Termodinâmica (Editora Livraria da Física, São Paulo, 2005).

[7] F. Reif, Fundamentals of statistical and thermal physics (McGraw-Hill, Singapore, 1965).

[8] P.T. Landsberg, Thermodynamics and statistical mechanics (Dover, New York, 1990).

[9] D.V. Schroeder, An introduction to thermal physics (Addison-Wesley-Longman, New York, 2000).

[10] S.R.A. Salinas, Introdução à física estatística (EDUSP, São Paulo, 2005).

[11] K. Huang, Introduction to statistical physics (CRC Press, New York, 2010), $2^{a}$ Ed.

[12] M.J. de Oliveira, Rev. Bras. Ens. Fis. 41, e20180174 (2019).

[13] S.S. Dourado e M.A. Marchiori, Rev. Bras. Ens. Fis. 41, e20180067 (2019).

[14] M.G. Calkin e D. Kiang, Am. J. Phys. 51, 78 (1983).

[15] J.S. Thomsen e H.C. Bers, Am. J. Phys. 64, 580 (1996).

[16] J.F. Stilck e R.M. Brum, Rev. Bras. Ens. Fis. 35, 4306 (2013).

[17] F.M.S. Lima, Rev. Bras. Ens. Fis. 37, 1701 (2015).

[18] W.H.C. Freire e D. Leite, Rev. Bras. Ens. Fis. 38, 1702 (2016).

[19] https://physicstoday.scitation.org/do/10.1063/ PT.6.40.20171009b/full/ 